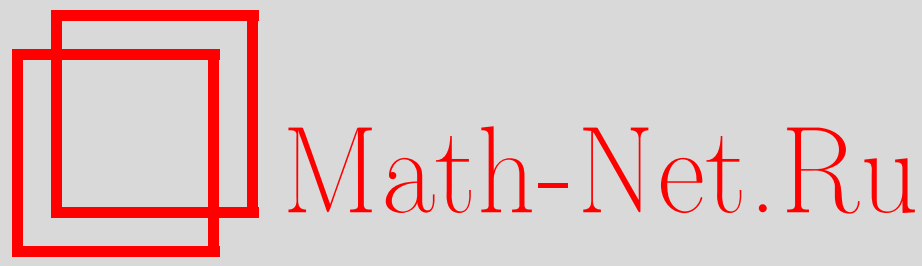

Я. М. Хусанбаев, Об асимптотике ветвящихся процессов с иммиграцией, Дискрет. матем., 2016, том 28, выпуск 1, 113-122

DOI: https://doi.org/10.4213/dm1361

Использование Общероссийского математического портала Math-Net.Ru подразумевает, что вы прочитали и согласны с пользовательским соглашением http://www . mathnet.ru/rus/agreement

Параметры загрузки:

IP : 3.80 .181 .102

26 апреля 2023 г., 16:39:12

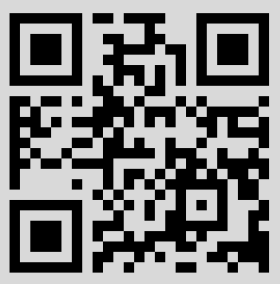




\title{
Об асимптотике ветвящихся процессов с иммиграцией
}

\author{
(ㄷ) 2016 г. .М. Хусанбаев*
}

Рассмотрена последовательность почти критических ветвящихся процессов с иммиграцией в случае, когда иммиграция образует стационарный процесс в широком смысле. Изучены скорость роста и асимптотика флуктуации таких процессов.

Ключевые слова: ветвящиеся процессы, иммиграция, стационарный процесс

\section{Введение и обозначения}

Пусть $\left\{\xi_{k, j}, k, j \in \mathbb{N}\right\}$ и $\left\{\varepsilon_{k}, k \in \mathbb{N}\right\}$ - две независимые совокупности независимых, неотрицательных, принимающих целые значения и одинаково распределенных случайных величин. Случайный процесс $X_{k}, k \geqslant 0$ определим следующими рекуррентными соотношениями:

$$
X_{0}=0, \quad X_{k}=\sum_{j=1}^{X_{k-1}} \xi_{k, j}+\varepsilon_{k}, \quad k \in \mathbb{N} .
$$

Обычно величину $X_{k}$ интерпретируют как общее число частиц в $k$-том поколении некоторой популяции, причем считается, что величина $\xi_{k, j}$ представляет собой число потомков $j$-той частицы $(k-1)$-го поколения популяции частиц, а величина $\varepsilon_{k}-$ число иммигрантов, поступивших в популяцию извне в $k$-м поколении.

Последовательность $\left\{X_{k}, k \geqslant 0\right\}$ называют ветвящимся процессом с иммиграцией. Предположим, что величины

$$
m=\mathbf{E} \xi_{1,1}, \quad \sigma^{2}=\mathbf{D} \xi_{1,1}, \quad \lambda=\mathbf{E} \varepsilon_{1}, \quad b^{2}=\mathbf{D} \varepsilon_{1}
$$

конечны. Ветвящийся процесс с иммиграцией $\left\{X_{k}, k \geqslant 0\right\}$ называют докритическим, критическим и надкритическим, если $m<1, m=1$ и $m>1$, соответственно. При изучении процессов вида (1) одной из важных и интересных задач является нахождение асимптотик распределения $X_{n}$ при $n \rightarrow \infty$. Если $X_{0}=1, \varepsilon_{k} \equiv 0, k \in \mathbb{N}$, то (1) представляет собой ветвящийся процесс Гальтона-Ватсона, и его асимптотика хорошо изучена (см., например, [1]).

*Место работы: Институт математики при Национальном Университете Узбекистана, e-mail: yakubjank@mail.ru 
В критическом случае E.Seneta [2] доказал, что распределение величины $n^{-1} X_{n}$ при $n \rightarrow \infty$ слабо сходится к гамма-распределению, a C.Z.Wei и J.Winnicki [3] установили, что ступенчатый случайный процесс $n^{-1} X_{[n t]}, t \geqslant 0$ при $n \rightarrow \infty$ слабо сходится в пространстве Скорохода $D[0, \infty)$ к решению стохастического дифференциального уравнения, где знак $[a]$ означает целую часть числа $a$. В работе [4] для критических, а в работе [5] для докритических ветвящихся процессов установлены функциональные предельные теоремы в случае, когда иммиграция является неоднородной. С.В. Нагаев [6] рассмотрел ветвящийся процесс с иммиграцией в случае, когда поток иммиграции образует стационарный в широком смысле процесс, и доказал, что распределение величины $n^{-1} X_{n}$ слабо сходится к гамма-распределению. Результат работы [6] был обобщен М.Х. Асадуллиным и С.В. Нагаевым [14] на случай, когда поток иммиграции $\varepsilon_{k}, k \geqslant 1$ такой, что существует случайная величина $\varepsilon$, для которой $n^{-1} \mathbf{E}\left|\sum_{k=1}^{n}\left(\varepsilon_{n}-\varepsilon\right)\right| \rightarrow 0$ при $n \rightarrow \infty$, т.е. последовательность $\varepsilon_{k}, k \geqslant 1$ обладает свойством "эргодичности”. Заслуживает внимания тот факт, что при этом не потребовалось ни независимости, ни одинаковой распределенности величин $\varepsilon_{k}$.

Пусть теперь при каждом $n \in \mathbb{N}\left\{\xi_{k, j}^{(n)}, k, j \in \mathbb{N}\right\}$ и $\left\{\varepsilon_{k}^{(n)}, k \in \mathbb{N}\right\}$ - две независимые совокупности независимых, неотрицательных целочисленных одинаково распределенных случайных величин. Определим последовательность ветвящихся процессов с иммиграцией $\left\{X_{k}^{(n)}, k \geqslant 0\right\}, n \in \mathbb{N}$, рекуррентными соотношениями

$$
X_{0}^{(n)}=0, \quad X_{k}^{(n)}=\sum_{j=1}^{X_{k-1}^{(n)}} \xi_{k, j}^{(n)}+\varepsilon_{k}^{(n)}, \quad k, n \in \mathbb{N} .
$$

Будем предполагать, что величины

$$
m_{n}=\mathbf{E} \xi_{1,1}^{(n)}, \quad \sigma_{n}^{2}=\mathbf{D} \xi_{1,1}^{(n)}, \quad \lambda_{n}=\mathbf{E} \varepsilon_{1}^{(n)}, \quad b_{n}^{2}=\mathbf{D} \varepsilon_{1}^{(n)}
$$

являются конечными для каждого $n \in \mathbb{N}$. Последовательность ветвящихся процессов $\left\{X_{k}^{(n)}, k \geqslant 0\right\}, n \in \mathbb{N}$ называют почти критической, если $m_{n} \rightarrow 1$ при $n \rightarrow \infty$.

В [7] T.N. Sriram рассмотрел случай, когда $m_{n}=1+\alpha n^{-1}+o\left(n^{-1}\right)(\alpha \in \mathbb{R})$, $\sigma_{n}^{2} \rightarrow \sigma^{2}>0$ при $n \rightarrow \infty$, и установил, что процесс $n^{-1} X_{[n t]}^{(n)}$ при $n \rightarrow \infty$ слабо сходится в $D[0, \infty)$ к решению стохастического дифференциального уравнения. В [8] M. Ispany, G. Pap, Van Zuijlen рассмотрели случай, когда $m_{n}=1+\alpha n^{-1}+o\left(n^{-1}\right)$ $(\alpha \in \mathbb{R}), \sigma_{n}^{2} \rightarrow 0$ при $n \rightarrow \infty$, и доказали, что процесс $n^{-1} X_{[n t]}^{(n)}$ при $n \rightarrow \infty$ слабо сходится в $D[0, \infty)$ к неслучайной функции, а также установили, что процесс $n^{-1 / 2}\left(X_{[n t]}^{(n)}-\mathbf{E} X_{[n t]}^{(n)}\right)$ при $n \rightarrow \infty$ слабо сходится в $D[0, \infty)$ к решению стохастического дифференциального уравнения, а в [13] исследована скорость сходимости одномерных распределений $n^{-1 / 2}\left(X_{[n t]}^{(n)}-\mathbf{E} X_{[n t]}^{(n)}\right)$ к предельному закону. В [9] установлены функциональные предельные теоремы для почти критических ветвящихся процессов с иммиграцией при различных условиях на числовые характеристики величин $\xi_{1,1}^{(n)}$ и $\varepsilon_{1}^{(n)}$. В [15] И.С. Бадалбаев и А.М. Зубков рассмотрели последовательность специально построенных случайных (не обязательно ветвящихся) процессов и доказали предельную теорему (теорема 1), которая содержит результаты работ [6] и [14] как частный случай. 
Здесь рассматривается последовательность почти критических ветвящихся процессов с иммиграцией в случае, когда поток иммиграции образует стационарный процесс в широком смысле. Исследована асимптотика таких процессов и получена асимптотическая формула для характеристической функции его флуктуации.

Пусть при каждом $n \in \mathbb{N}\left\{\tau_{k}^{(n)}, k \in \mathbb{N}\right\}$ - стационарный в широком смысле процесс, причем случайные величины $\tau_{k}^{(n)}$ неотрицательны и принимают целые значения. Предположим, что при каждом $n \in \mathbb{N}$ совокупность случайных величин $\left\{\xi_{k, j}^{(n)}, k, j \in \mathbb{N}\right\}$ и процесс $\left\{\tau_{k}^{(n)}, k \in \mathbb{N}\right\}$ являются независимыми. Определим последовательность ветвящихся процессов с иммиграцией $\left\{Z_{k}^{(n)}, k \in \mathbb{N}\right\}, n \in \mathbb{N}$, рекуррентными соотношениями

$$
Z_{0}^{(n)}=0, \quad Z_{k}^{(n)}=\sum_{j=1}^{Z_{k-1}^{(n)}} \xi_{k, j}^{(n)}+\tau_{k}^{(n)}, k, n \in \mathbb{N} .
$$

Обозначим через $Y_{n, i}^{j}(k), k=i, i+1, \ldots$, ветвящийся процесс Гальтона-Ватсона, порожденный $j$-й из поступивших в момент $i$ частиц в $n$-й серии. В силу сделанных предположений при каждом $n \in \mathbb{N}$ ветвящиеся процессы $\left\{Y_{n, i}^{j}(k), k=i, i+1, \ldots\right\}$, $j, i \geqslant 1$ независимы, причем $Y_{n, i}^{j}(k+i), k=0,1, \ldots$, имеют такое же распределение, как и $Y_{n, 1}^{1}(k), k=1,2, \ldots$

Введем в рассмотрение случайные ступенчатые функции $Z_{n}(t), t \geqslant 0, n \in \mathbb{N}$, определенные соотношением $Z_{n}(t)=Z_{[n t]}^{(n)}, t \geqslant 0$. Положим

$$
\begin{gathered}
\gamma_{n}=\mathbf{E} \tau_{1}^{(n)}, \quad b_{n}=\mathbf{D} \tau_{1}^{(n)}, \quad \rho_{n}(k)=\operatorname{cov}\left(\tau_{1}^{(n)}, \tau_{k+1}^{(n)}\right), \\
\varphi_{n, k}(z)=\mathbf{E} e^{i z Y_{n, 1}^{1}(k)}, \quad \Phi_{n}(z, t)=\mathbf{E} e^{i z Z_{n}(t)}
\end{gathered}
$$

Обозначим через $\varepsilon_{n, k}(z)$ остаточный член в представлении $\varphi_{n, k}(z)$ по формуле Тейлора для характеристических функций

$$
\varphi_{n, k}(z)=1+i z \mathbf{E} Y_{n, 1}^{1}(k)-\frac{z^{2}}{2} \mathbf{E}\left[Y_{n, 1}^{1}(k)\right]^{2}+\frac{z^{2}}{2} \varepsilon_{n, k}(z), \quad k, n \in \mathbb{N} .
$$

Известно ([11], стр. 297, теорема 1), что $\varepsilon_{n, k}(z) \rightarrow 0$ при $z \rightarrow 0$ и $\left|\varepsilon_{n, k}(z)\right| \leqslant$ $3 \mathbf{E}\left[Y_{n, 1}^{1}(k)\right]^{2}$. В дальнейшем всюду знак $\stackrel{P}{\longrightarrow}$ будет обозначать сходимость по вероятности случайных величин.

\section{Формулировка результатов}

Следующая теорема дает представление об асимптотическом поведении процесса $Z_{n}(t), t \geqslant 0$.

Теорема 1. Пусть выполнены следующие условия:

A) $m_{n}=1+\frac{\alpha}{n}+o\left(n^{-1}\right)$ при $n \rightarrow \infty$ для некоторого $\alpha \in \mathbb{R}$;

B) $\sigma_{n}^{2} \rightarrow 0$ npu $n \rightarrow \infty$;

C) $\gamma_{n} \rightarrow \gamma \geqslant 0, b_{n}^{2} \rightarrow b^{2} \geqslant 0$ npu $n \rightarrow \infty$;

D) $\frac{1}{n} \sum_{k=1}^{n}\left|\rho_{n}(k)\right| \rightarrow 0$ nрu $n \rightarrow \infty$.

Тогда имеет место слабая сходимость $\frac{Z_{n}}{n} \rightarrow \mu$ при $n \rightarrow \infty$ в пространстве $D[0, \infty)$, где $\mu$ определяется соотношением $\mu(t)=\gamma \int_{0}^{t} e^{\alpha u} d u$. 
Из теоремы 1 и из основной теоремы [10] легко получить утверждение о слабой сходимости для общего числа частиц в ветвящемся процессе с иммиграцией.

Следствие 1. Пусть выполнены условия теоремы 1. Тогда имеет место слабая сходимость $\frac{1}{n^{2}} \sum_{k=1}^{[n t]} Z_{k}^{(n)} \rightarrow \int_{0}^{t} \mu(s) d s$ nри $n \rightarrow \infty$ в пространстве $D[0, \infty)$, где $\mu$ - прочесс из теоремы 1.

Замечание 1. Если величины $\tau_{k}^{(n)}, k \in \mathbb{N}$, независимы или обладают "свойством мартингальности", т.е. если

$$
\mathbf{E}\left(\tau_{k}^{(n)}-\gamma_{n} \mid \tau_{1}^{(n)}, \ldots, \tau_{k-1}^{(n)}\right)=0, \quad k \geqslant 2,
$$

то условие $\mathbf{D}$ выполнено, и полученный в теореме 1 результат согласуется с результатом теоремы $2.1[8]$.

Замечание 2. Так как $\left|\rho_{n}(k)\right| \leqslant b_{n}^{2}$, то условие $\mathbf{D}$ имеет место, если $b_{n}^{2} \rightarrow 0$ при $n \rightarrow \infty$.

Следующая теорема дает представление об асимптотике величины $n^{-1 / 2}\left(Z_{n}^{(n)}-\mathbf{E} Z_{n}^{(n)}\right)$.

Теорема 2. Пусть выполнены условия $\boldsymbol{A}, \boldsymbol{C}$ и $\boldsymbol{D}$ теоремы 1 и следующие условия: $\mathbf{B}_{1} \cdot n \sigma_{n}^{2} \rightarrow \sigma^{2}>0$ npu $n \rightarrow \infty$;

E. $\frac{1}{n} \sum_{k=1}^{n}\left|\varepsilon_{n, k}\left(\frac{z}{\sqrt{n}}\right)\right| \rightarrow 0$ при $n \rightarrow \infty$ для любого $z>0$.

Тогда имеет место асимптотическое соотношение

$$
\mathbf{E} e^{i z \frac{Z_{n}^{(n)}-\mathbf{E} Z_{n}^{(n)}}{\sqrt{n}}}=e^{-\frac{z^{2}}{2} \cdot \frac{\gamma \sigma^{2}\left(e^{\alpha}-1\right)^{2}}{2 \alpha^{2}}} \mathbf{E} e^{i z \frac{1}{\sqrt{n}} \sum_{k=1}^{n} m_{n}^{n-k} \cdot\left(\tau_{k}^{(n)}-\gamma_{n}\right)}+o(1) n p u n \rightarrow \infty .
$$

Из теоремы 2 и из замечания 2 получаем следующее утверждение.

Следствие 2. Если выполнены условия $\boldsymbol{A}, \boldsymbol{C}$ теоремы 1 и условия $\mathbf{B}_{1}, \boldsymbol{E}$ теоремь 2 и при этом $n b_{n}^{2} \rightarrow 0$, то распределение случайной величинь $n^{1 / 2}\left(Z_{n}^{(n)}-\mathbf{E} Z_{n}^{(n)}\right)$ слабо сходится $\kappa$ нормальному закону со средним нуль и дисперсией $\frac{\gamma \sigma^{2}\left(e^{\alpha}-1\right)^{2}}{2 \alpha^{2}}$.

Замечание 3. Если $\tau_{k}^{(n)}, k \in \mathbb{N}$, является стационарным в узком смысле процессом, то асимптотику характеристической функции $n^{-1 / 2} \sum_{k=1}^{n} m_{n}^{n-k}\left(\tau_{k}^{(n)}-\gamma_{n}\right)$ можно найти с помощью известных результатов для стационарных в узком смысле случайных процессов. Если $\tau_{k}^{(n)}, k \in \mathbb{N}$, является однородной цепью Маркова, то можно воспользоваться результатами из [12].

Теорема 3. Пусть при каждом $n \in \mathbb{N}$ случайные величины $\tau_{k}^{(n)}, k \in \mathbb{N}$, независимъ и одинаково распределены и пусть выполнены условия А, С теоремы 1, $\mathbf{B}_{1}$ и $\boldsymbol{E}$ теоремы 2. Если, кроме того,

$$
\mathbf{E}\left(\tau_{1}^{(n)}-\gamma_{n}\right)^{2} I\left(\left|\tau_{1}^{(n)}-\gamma_{n}\right|>\varepsilon \sqrt{n}\right) \rightarrow 0 \text { npu } n \rightarrow \infty,
$$


то распределение случайной величинь $n^{-1 / 2}\left(Z_{n}^{(n)}-\mathbf{E} Z_{n}^{(n)}\right)$ слабо сходится $\kappa$ нормальному закону со средним нуль и дисперсией $\frac{\left(e^{\alpha}-1\right)^{2}}{2 \alpha^{2}} \gamma \sigma^{2}+\frac{b^{2}\left(e^{2 \alpha}-1\right)}{2 \alpha}$.

Замечание 4. Теорема 3 согласуется с одномерным вариантом теоремы 2.2 из [8].

Примеры. Приведем примеры случайных величин $\xi_{k, j}^{(n)}$ и $\tau_{k}^{(n)}$, для которых выполнены условия теорем 1 и 2 .

1. Пусть случайная величина $\xi_{k, j}^{(n)}$ принимает значения 0,1 и 2 с вероятностями $0,75 n^{-1}, 1-n^{-1}$ и $0,25 n^{-1}$ соответственно, а $\left\{\tau_{k}^{(n)}, k \in \mathbb{N}\right\}$ - стационарная цепь Маркова с конечным пространством состояний $E=\{0,1,2, \ldots, l\}$, неприводимая и апериодичная. Имеем $m=1-0,5 n^{-1}, \sigma_{n}^{2}=n^{-1}\left(1-0,25 n^{-1}\right)$. Следовательно, выполнены условия $\mathbf{A}, \mathbf{B}$ теоремы 1 и условие $\mathbf{B}_{1}$ теоремы 2. Разлагая $\varphi_{n, k}(z)$ в ряд Тейлора и учитывая лемму 2 из [13], нетрудно убедиться в том, что условие $\mathbf{E}$ теоремы 2 выполнено. Далее, из теории цепей Маркова известно, что цепь $\left\{\tau_{k}^{(n)}, k \in \mathbb{N}\right\}$ удовлетворяет условию $\varphi$-перемешивания, причем существует такое число $0<a_{n}<1$, что коэффициент перемешивания убывает как геометрическая прогрессия $a_{n}^{k}$ при $k \rightarrow \infty$. Поэтому, применяя лемму 1 ([10], стр. 236), имеем $\left|\rho_{n}(k)\right| \leqslant 2 a_{n}^{k / 2} b_{n}^{2}$. Если, кроме того, существуют конечные пределы $a_{n} \rightarrow a, \gamma_{n} \rightarrow \gamma$, $b_{n}^{2} \rightarrow b^{2}$ при $n \rightarrow \infty$, причем $0 \leqslant a<1$, то выполнены условия $\mathbf{C}$ и $\mathbf{D}$. Значит, в рассматриваемом случае выполнены все условия теорем 1 и 2 .

2. Пусть $\xi_{1,1}^{(n)}$ имеет геометрическое распределение:

$$
P\left(\xi_{1,1}^{(n)}=0\right)=p_{n}, \quad P\left(\xi_{1,1}^{(n)}=k\right)=\left(1-p_{n}\right)^{2} p_{n}^{k-1}, \quad k=1,2, \ldots,
$$

где $p_{n} \in(0,1)$ имеет вид $p_{n}=\frac{\alpha}{n}+o\left(\frac{1}{n}\right)$. Здесь $\alpha>0-$ фиксированное число. Пусть величина $\tau_{k}^{(n)}$ имеет распределение Бернулли с параметром $r_{n}$, причем $r_{n}=$ $1-\frac{\beta}{n}+o\left(\frac{1}{n}\right)$, где $\beta \geqslant 0-$ фиксированное число. Имеем $m_{n}=1, \sigma_{n}^{2}=\frac{2 \alpha}{n}+o\left(\frac{1}{n}\right)$, $\mathbf{E} \xi_{1,1}^{(n)}\left(\xi_{1,1}^{(n)}-1\right)\left(\xi_{1,1}^{(n)}-2\right)=\frac{6 \alpha^{2}}{n^{2}}+o\left(\frac{1}{n^{2}}\right), \gamma_{n}=1-\frac{\beta}{n}+o\left(\frac{1}{n}\right), b_{n}^{2}=\frac{\beta}{n}+o\left(\frac{1}{n}\right)$

$$
\mathbf{E}\left(Y_{n, 1}^{1}(k)\right)^{3}=\frac{6\left(k p_{n}\right)^{2}+6 k p_{n}\left(1-p_{n}\right)+\left(1-p_{n}\right)^{2}}{\left(1-p_{n}\right)^{2}} .
$$

Следовательно, в рассматриваемом случае условия $\mathbf{A}, \mathbf{B}, \mathbf{B}_{1}$ и $\mathbf{E}$ выполнены. Очевидно, что условие $\mathbf{C}$ выполнено. Далее, условие $\mathbf{D}$ выполнено в силу того, что $\left|\rho_{n}(k)\right| \leqslant p_{n}\left(1-p_{n}\right) \leqslant \frac{2 \beta}{n}$ для достаточно больших $n$. Значит, выполнены все условия теорем 1 и 2 .

\section{Доказательства теорем}

Доказательство теоремы 1. Ясно, что случайную величину $Z_{k}^{(n)}$ можно представить в виде

$$
Z_{k}^{(n)}=\sum_{i=1}^{k} \sum_{j=1}^{\tau_{i}^{(n)}} Y_{n, i}^{j}(k) .
$$

Поэтому, учитывая независимость величин $Y_{n, i}^{j}(k)$, имеем

$$
\Phi_{n}(z, t)=\mathbf{E} \prod_{k=1}^{[n t]} \varphi_{n,[n t]-k}^{\tau_{k}^{(n)}}(z)=\mathbf{E} \prod_{k=1}^{[n t]} \varphi_{n, k}^{\tau_{[n]-k}^{(n)}}(z) .
$$


Рассмотрим только случай, когда $\alpha \neq 0$. Хорошо известно, что

$$
\mathbf{E} Y_{n, 1}^{1}(k)=m_{n}^{k}, \quad \mathbf{E}\left[Y_{n, 1}^{1}(k)\right]^{2}=\frac{m_{n}^{k-1}\left(m_{n}^{k}-1\right)}{m_{n}-1} \sigma_{n}^{2}+m_{n}^{2 k} .
$$

Так как $\ln x=x-1+O\left((x-1)^{2}\right)$, то, учитывая (4) и (6), имеем

$$
\begin{gathered}
\ln \prod_{k=1}^{[n t]} \varphi_{n,[n t]-k}^{\tau_{k}^{(n)}}\left(\frac{z}{n}\right)=\frac{i z}{n} \sum_{k=1}^{[n t]} m_{n}^{[n t]-k} \tau_{k}^{(n)}- \\
-\frac{z^{2}}{2 n^{2}} \sum_{k=1}^{[n t]} \tau_{k}^{(n)}\left\{\frac{m_{n}^{[n t]-k-1}\left(m_{n}^{[n t]-k}-1\right)}{m_{n}-1} \sigma_{n}^{2}+m_{n}^{2([n t]-k)}\right\}+ \\
+\frac{z^{2}}{2 n^{2}} \sum_{k=1}^{[n t]} \tau_{k}^{(n)} \varepsilon_{n,[n t]-k}\left(\frac{z}{n}\right)+O\left(\frac{z^{2}}{n^{2}} \sum_{k=1}^{[n t]} m_{n}^{2([n t]-k)} \tau_{k}^{(n)}\right)= \\
=i z I_{n}-\frac{z^{2}}{2}\left(J_{n}+K_{n}+M_{n}\right) .
\end{gathered}
$$

Лемма 1. Имеет место следующее:

1) если $m_{n} \neq 1$, то $\mathbf{E} Z_{k}^{(n)}=\frac{m_{n}^{k}-1}{m_{n}-1} \gamma_{n}$, и если $m_{n}=1$, mo $\mathbf{E} Z_{k}^{(n)}=k \gamma_{n}$;

2) если $m_{n} \neq 1$, mo

$$
\begin{aligned}
\mathbf{D} Z_{k}^{(n)}= & \frac{\left(m_{n}^{k-1}-1\right)\left(m_{n}^{k}-1\right)}{\left(m_{n}-1\right)\left(m_{n}^{2}-1\right)} \gamma_{n} \sigma_{n}^{2}+\frac{m_{n}^{2 k-1}-1}{m_{n}^{2}-1} b_{n}^{2}+ \\
& +2 \sum_{i=1}^{k} \sum_{j=i+1}^{k} \rho_{n}(j-i) m_{n}^{2 n-j-i} ;
\end{aligned}
$$

если $m_{n}=1$, mo

$$
\mathbf{D} Z_{k}^{(n)}=\frac{k(k-1)}{2} \gamma_{n} \sigma_{n}^{2}+k b_{n}^{2}+2 \sum_{i=1}^{k}(k-i) \rho(i) .
$$

Доказательство леммы несложно. Поэтому мы его опускаем.

Рассмотрим $I_{n}$. Ясно, что

$$
\mathbf{E} I_{n}=\gamma_{n} \frac{m_{n}^{[n t]}-1}{n\left(m_{n}-1\right)} \rightarrow \gamma \frac{e^{\alpha t}-1}{\alpha} \text { при } n \rightarrow \infty .
$$

Учитывая условия теоремы имеем

$$
\begin{aligned}
\mathbf{D} I_{n} & =\frac{b_{n}^{2}}{n^{2}} \cdot \frac{m_{n}^{2[n t]}-1}{m_{n}^{2}-1}+\frac{2}{n^{2}} \sum_{k=1}^{[n t]} \sum_{j=k+1}^{[n t]} \rho_{n}(j-k) m_{n}^{2[n t]-k-j} \leqslant \\
& \leqslant 2 \frac{b_{n}^{2}}{n} \cdot \frac{e^{2 \alpha t}-1}{2 \alpha}+2 \max \left(1, m_{n}^{n}\right) \cdot \frac{1}{n} \sum_{k=1}^{n}\left|\rho_{n}(k)\right|+o(1) \rightarrow 0
\end{aligned}
$$

при $n \rightarrow \infty$. Отсюда, из (8) и неравенства Чебышева получаем:

$$
I_{n} \stackrel{P}{\rightarrow} \gamma \frac{e^{\alpha t}-1}{\alpha} \text { при } n \rightarrow \infty .
$$


Теперь рассмотрим $J_{n}$. Нетрудно видеть, что

$$
\mathbf{E} J_{n}=\frac{\gamma_{n}}{2 \alpha}\left(\frac{\sigma_{n}^{2}}{\alpha m_{n}}\left(e^{\alpha t}-1\right)^{2}+\frac{e^{2 \alpha t}-1}{n}\right)+o(1) \rightarrow 0 \text { при } n \rightarrow \infty .
$$

Поскольку $J_{n} \geqslant 0$ с вероятностью 1 , то из последнего соотношения следует, что

$$
J_{n} \stackrel{P}{\longrightarrow} 0 \text { при } n \rightarrow \infty .
$$

Далее, так как $\left|\varepsilon_{n, k}(t)\right| \leqslant 3 \mathbf{E}\left[Y_{n, 1}^{1}(k)\right]^{2}$, то аналогично (10) получаем

$$
K_{n} \stackrel{P}{\longrightarrow} 0 \text { при } n \rightarrow \infty .
$$

Рассуждения, аналогичные проведенным при выводе (9), доказывают, что

$$
\frac{1}{n^{2}} \sum_{k=1}^{[n t]} m_{n}^{2([n t]-k)} \tau_{k}^{(n)} \stackrel{P}{\longrightarrow} 0 .
$$

Теперь отсюда и из (9)-(11) следует, что

$$
\ln \prod_{k=1}^{[n t]} \varphi_{n,[n t]-k}^{\tau_{k}^{(n)}}\left(\frac{z}{n}\right) \stackrel{P}{\longrightarrow} i z \gamma \frac{e^{\alpha t}-1}{\alpha} \text { при } n \rightarrow \infty .
$$

Тогда в силу теоремы Лебега о мажорируемой сходимости

$$
\Phi_{n}\left(\frac{z}{n}, t\right) \rightarrow e^{i z \gamma \frac{e^{\alpha t}-1}{\alpha}} \text { при } n \rightarrow \infty .
$$

Значит, для любого $t \geqslant 0$

$$
\frac{Z_{n}(t)}{n} \stackrel{P}{\longrightarrow} \gamma \frac{e^{\alpha t}-1}{\alpha} \text { при } n \rightarrow \infty .
$$

Теперь, применяя известное неравенство

$$
\left|\prod_{i=1}^{k} a_{i}-\prod_{i=1}^{k} b_{i}\right| \leqslant \sum_{i=1}^{k}\left|a_{i}-b_{i}\right|, \quad\left|a_{i}\right| \leqslant 1, \quad\left|b_{i}\right| \leqslant 1,
$$

теорему Лебега о мажорируемой сходимости и учитывая (12), приходим к выводу, что конечномерные распределения процесса $n^{-1} Z_{n}(t), t \geqslant 0$, сходятся по вероятности к конечномерным распределениям $\mu$.

Так как предельный процесс непрерывен, то для завершения доказательства теоремы остается доказать плотность семейства $\left\{n^{-1} Z_{n}(t), t \in[0, T]\right\}$ при любом $T>0$. Нетрудно видеть, что

$$
\mathbf{E} \frac{Z_{n}(t)}{n}=\frac{m_{n}^{[n t]}-1}{m_{n}-1} \gamma_{n} \rightarrow \frac{e^{\alpha t}-1}{\alpha} \gamma \text { при } n \rightarrow \infty .
$$

Теперь, применяя лемму, согласно условиям теоремы имеем

$$
\mathbf{E}\left(\frac{Z_{n}(t)}{n}-\frac{Z_{n}(s)}{n}\right)^{2} \leqslant \frac{3}{n^{2}}\left[\left(\mathbf{D} Z_{n}(t)+\mathbf{D} Z_{n}(s)\right)+\left(\mathbf{E} Z_{n}(t)-\mathbf{E} Z_{n}(s)\right)^{2}\right]=
$$




$$
\begin{gathered}
=3\left(\frac{\left(e^{\alpha t}-1\right)^{2}}{2 \alpha^{2}}+\frac{\left(e^{\alpha s}-1\right)^{2}}{2 \alpha^{2}}\right) \gamma_{n} \sigma_{n}^{2}+\frac{3 b_{n}^{2}}{n} \cdot \frac{e^{2 \alpha t}+e^{2 \alpha s}-2}{2 \alpha}+ \\
+4 t \max \left(1, e^{\alpha t}\right) \cdot \frac{1}{n} \sum_{k=1}^{[n t]} \rho_{n}(k)+\frac{3 \gamma^{2}}{|\alpha|} \max \left(1, e^{\alpha t}\right)(t-s)^{2}+o(1)
\end{gathered}
$$

для любых $0<s<t<T$ и для достаточно больших $n$. Следовательно, для достаточно больших $n$

$$
\mathbf{E}\left(\frac{1}{n} Z_{[n t]}^{(n)}-\frac{1}{n} Z_{[n s]}^{(n)}\right)^{2} \leqslant 8 \frac{\gamma^{2}}{|\alpha|} e^{2|\alpha| T}(t-s)^{2} .
$$

Тогда согласно критерию плотности (теорема 12.3 [10]) последовательность процессов $\left\{n^{-1} Z_{n}(t), t \in[0, T]\right\}$ является плотной. Доказательство теоремы 1 завершено.

Замечание 5. Из доказательства теоремы 1 видно, что в условии $\mathbf{C}$ вместо условия $b_{n}^{2} \rightarrow b^{2}$ достаточно потребовать, чтобы $n^{-1} b_{n}^{2} \rightarrow 0$.

Доказательство теоремъ 2. Имеем

$$
\psi_{n}(z)=\mathbf{E} e^{i z \frac{z_{n}^{(n)}-\mathbf{E} Z_{n}^{(n)}}{\sqrt{n}}}=e^{-i z \frac{\mathbf{E} Z_{n}^{(n)}}{\sqrt{n}}} \Phi_{n}\left(\frac{z}{\sqrt{n}}, 1\right) .
$$

Так как

$$
\mathbf{E} Z_{n}^{(n)}=\frac{m_{n}^{n}-1}{m_{n}-1} \gamma_{n}=\sum_{k=1}^{n} m_{n}^{n-k} \gamma_{n}
$$

то

$$
\psi_{n}(z)=e^{-\frac{i z}{\sqrt{n}} \sum_{k=1}^{n} m_{n}^{n-k} \gamma_{n}} \mathbf{E} \prod_{k=1}^{n} \varphi_{n, n-k}^{\tau_{k}^{(n)}}\left(\frac{z}{\sqrt{n}}\right) .
$$

Учитывая разложение $\ln x=(x-1)-\frac{1}{2}(x-1)^{2}+O\left((x-1)^{3}\right)$ и соотношение $(4)$, имеем

$$
\begin{gathered}
\ln \left[e^{-\frac{i z}{\sqrt{n}} \sum_{k=1}^{n} m_{n}^{n-k} \gamma_{n}} \prod_{k=1}^{n} \varphi_{n, n-k}^{\tau_{k}^{(n)}}\left(\frac{z}{\sqrt{n}}\right)\right]= \\
=\frac{i z}{\sqrt{n}} \sum_{k=1}^{n} m_{n}^{n-k}\left(\tau_{k}^{(n)}-\gamma_{n}\right)-\frac{z^{2}}{2 n} \sum_{k=1}^{n} \tau_{k}^{(n)} \frac{m_{n}^{n-k}\left(m_{n}^{n-k}-1\right)}{m_{n}\left(m_{n}-1\right)} \sigma_{n}^{2}+ \\
+O\left(\frac{z^{2}}{2 n} \sum_{k=1}^{n} \tau_{k}^{(n)} \varepsilon_{n, n-k}\left(\frac{z}{\sqrt{n}}\right)+\frac{|z|^{3}}{n \sqrt{n}} \sum_{k=1}^{n} \tau_{k}^{(n)} m_{n}^{3(n-k)}\right)=I_{1}+I_{2}+O\left(I_{3}\right) .
\end{gathered}
$$

Рассмотрим $I_{2}$. Ясно, что

$$
\begin{gathered}
\mathbf{E} I_{2}=-\frac{z^{2}}{2 n} \gamma_{n} \sum_{k=1}^{n} \frac{m_{n}^{n-k}\left(m_{n}^{n-k}-1\right)}{m_{n}\left(m_{n}-1\right)} \sigma_{n}^{2}= \\
=-\frac{z^{2} \gamma_{n}}{2 \alpha} \cdot \frac{\left(e^{\alpha}-1\right)^{2}}{2 \alpha}+o(1) \rightarrow-\frac{z^{2} \gamma}{4 \alpha^{2}}\left(e^{\alpha}-1\right)^{2} \sigma^{2} .
\end{gathered}
$$

Оценим дисперсию величины $I_{2}$. Учитывая лемму, имеем

$$
\mathbf{D} I_{2}=\frac{z^{4} b_{n}^{2}}{4 n^{2}} \sum_{k=1}^{n} \frac{m_{n}^{2(n-k)}\left(m_{n}^{n-k}-1\right)^{2}}{m_{n}^{2}\left(m_{n}-1\right)^{2}} \sigma_{n}^{4}+
$$




$$
\begin{gathered}
+\frac{z^{4}}{2 n^{2}} \sum_{k=1}^{n} \sum_{j=k+1}^{n} \rho_{n}(j-k) \frac{m_{n}^{2 n-k-j}\left(m_{n}^{n-k}-1\right)\left(m_{n}^{n-j}-1\right)}{m_{n}^{2}\left(m_{n}-1\right)^{2}} \sigma_{n}^{4} \leqslant \\
\leqslant \frac{z^{4}\left(n \sigma_{n}^{2}\right)^{2} \max \left(1, m_{n}^{4 n}\right)}{4 \alpha^{2} m_{n}^{2} n}\left(b_{n}^{2}+2 \sum_{k=1}^{n} \rho_{n}(k)\right) \rightarrow 0 \text { при } n \rightarrow \infty
\end{gathered}
$$

согласно условиям теоремы. Отсюда и из (15) следует, что

$$
I_{2} \stackrel{P}{\rightarrow} \frac{\gamma \sigma^{2}}{4 \alpha^{2}}\left(e^{\alpha}-1\right)^{2} .
$$

Далее,

$$
\mathbf{E}\left|I_{3}\right| \leqslant \frac{z^{2} \gamma_{n}}{2} \cdot \frac{1}{n} \sum_{k=1}^{n}\left|\varepsilon_{n, k}\left(\frac{z}{\sqrt{n}}\right)\right|+\frac{\gamma_{n}|z|^{3}}{n \sqrt{n}} \cdot \frac{m_{n}^{3[n t]}-1}{m_{n}^{3}-1} \rightarrow 0 \text { при } n \rightarrow \infty .
$$

Поэтому ясно, что $I_{3} \stackrel{P}{\longrightarrow} 0$ при $n \rightarrow \infty$. Отсюда и из $(14),(16)$ следует, что

$$
\begin{gathered}
\ln \left[e^{-\frac{i z}{\sqrt{n}} \sum_{k=1}^{n} m_{n}^{n-k} \gamma_{n}} \prod_{k=1}^{n} \varphi_{n, n-k}^{\tau_{k}^{(n)}}\left(\frac{z}{\sqrt{n}}\right)\right]= \\
=\frac{i z}{\sqrt{n}} \sum_{k=1}^{n} m_{n}^{n-k}\left(\tau_{k}^{(n)}-\gamma_{n}\right)-\frac{z^{2}}{4 \alpha^{2}}\left(e^{\alpha}-1\right)^{2} \gamma \sigma^{2}+o_{p}(1),
\end{gathered}
$$

где $o_{p}(1) \stackrel{P}{\longrightarrow} 0$ при $n \rightarrow \infty$. Отсюда и из теоремы Лебега о мажорируемой сходимости следует утверждение теоремы 2 .

Доказательство теоремы 3. Имеем

$$
\mathbf{E}\left(\frac{1}{\sqrt{n}} \sum_{k=1}^{n} m_{n}^{n-k}\left(\tau_{k}^{(n)}-\gamma_{n}\right)\right)^{2}=\frac{b_{n}^{2}}{n} \cdot \frac{m_{n}^{2 n}-1}{m_{n}^{2}-1} \rightarrow \frac{b^{2}\left(e^{2 \alpha}-1\right)}{2 \alpha} \text { при } n \rightarrow \infty .
$$

Так как величины $\tau_{k}^{(n)}, k=1,2, \ldots$, одинаково распределены, то

$$
\begin{aligned}
& \frac{1}{n} \sum_{k=1}^{n} m_{n}^{2(n-k)} \mathbf{E}\left(\tau_{k}^{(n)}-\gamma_{n}\right)^{2} I\left(m_{n}^{n-k}\left|\tau_{k}^{(n)}-\gamma_{n}\right|>\varepsilon \sqrt{n}\right) \leqslant \\
\leqslant & \max \left(1, m_{n}^{2 n}\right) \mathbf{E}\left(\tau_{1}^{(n)}-\gamma_{n}\right)^{2} I\left(\max \left(1, m_{n}^{n}\right)\left|\tau_{1}^{(n)}-\gamma_{n}\right|>\varepsilon \sqrt{n}\right) \rightarrow 0
\end{aligned}
$$

при $n \rightarrow \infty$. Поэтому согласно центральной предельной теореме получаем

$$
\mathbf{E} e^{\frac{i z}{\sqrt{n}} \sum_{k=1}^{n} m_{n}^{n-k}\left(\tau_{k}^{(n)}-\gamma_{n}\right)} \rightarrow e^{-\frac{z^{2}}{2} \cdot \frac{b^{2}\left(e^{2 \alpha}-1\right)}{2 \alpha}} \text { при } n \rightarrow \infty .
$$

Отсюда и из теоремы 2 следует утверждение теоремы 3.

Замечание 6. Если $\mathbf{E}\left|\xi_{1,1}^{(n)}\right|^{3}<\infty$ для всех $n \in \mathbb{N}$, то теорема 2 верна, если условие $\mathbf{E}$ заменить условием $\sqrt{n} \omega_{n} \rightarrow 0$ при $n \rightarrow \infty$, где $\omega_{n}$ - третий факториальный момент величины $\xi_{1,1}^{(n)}$.

Автор благодарит рецензента за замечания, которые способствовали улучшению рукописи. 


\section{Список литературы}

1. Athreya K.B., Ney P.E., Branching Processes, Springer-Verlag, New York, 1972, 288 pp.

2. Seneta E., "An explicit-limit theorem for the critical Galton-Watson process with Immigration", J. Roy. Stat. Soc., 32 (1970), 149-152.

3. Wei C.Z., Winicki J.," "Some asymptotic results for the branching process with immigration", Stoch. Process. Appl., 31 (1989), 261-282.

4. Rakhimov I., "Functional limit theorems for critical processes with immigration", Adv. Appl. Probab., 39 (2007), 1054-1069.

5. Хусанбаев Я.М., "Об асимптотике докритического ветвящегося процесса с иммиграцией”, Укр. мат. ж., 65:6 (2013), 835-843; англ. пер.:

6. Нагаев С.В., "Предельная теорема для ветвящихся процессов с иммиграцией", Теория вероятн. и ее применения, 20:1 (1975), 178-180; англ. пер.: Nagaev S. V., "A limit theorem for branching processes with immigration", Theory Probab. Appl., 20:1 (1975), 176-179.

7. Sriram T.N., "Invalidity of bootstrap for critical branching process with immigration", Ann. Statist., 22 (1994), 1013-1023.

8. Ispany M., Pap G. and Van Zuijlen M.C.A., "Fluctuation limit of branching processes with immigration and estimation of the mean", Adv. Appl. Probab., 37 (2005), 523-528.

9. Почти критические ветвящиеся процессы и предельные теоремы, Укр. матем. ж., 61:1 (2009), 127-133; англ. пер.: Khusanbaev Ya. M., "Almost critical branching processes and limit theorems", Ukr. Math. J., 61:1 (2009), 154-163.

10. Биллингсли П., Сходимость вероятностных мер, М.: Наука, 1977, 352 с.

11. Ширяев А.Н., Вероятность, М.: Наука, 1980, 575 с.; англ. пер.: Shiryaev А. N., 2, Graduate Texts in Mathematics, 95, Springer-Verlag, New York, 1996, 624 pp.

12. Сираждинов С.Х., Форманов Ш.К., Предельные теоремы для сумм случайных векторов, связанных в цепъ Маркова, Ташкент: Фан, 1979, 171 с.

13. Хусанбаев Я.М., "О скорости сходимости в одной предельной теореме для ветвящихся процессов с иммиграцией”, Сиб. матем. ж., 55:1 (2014), 221-227; англ. пер.: Khusanbaev Ya. M., "On the convergence rate in one limit theorem for branching processes with immigration", Siberian Math. J., 55:1 (2014), 178-184.

14. Асадуллин М.Х., Нагаев С.В., "Предельные теоремы для критического ветвящегося процесса с иммиграцией", Матем.заметки, 32:4 (1982), 537-548; англ. пер.: Asadullin M. Kh., Nagaev S. V., "Limit theorems for a critical branching process with immigration", Math. Notes, 32:4 (1982), 750-757.

15. Бадалбаев И.С., Зубков А.М., "Предельные теоремы для последовательности ветвящихся процессов с иммиграцией", Теория вероятностей и ее приложения, 28:2 (1983), 382-388; англ. пер.: Badalbaev I. S., Zubkov A. M., "Limit theorems for a sequence of branching processes with immigration", Theory Probab. Appl., 28:2 (1984), 404-409. 